\title{
Outage performance users located outside D2D coverage area in downlink cellular network
}

\author{
Thanh-Luan Nguyen, Dinh-Thuan Do \\ Faculty of Electronics Technology, Industrial University of Ho Chi Minh City (IUH), Ho Chi Minh City, Vietnam
}

\begin{tabular}{l}
\hline \hline Article Info \\
\hline Article history: \\
Received May 24, 2020 \\
Revised Apr 4, 2021 \\
Accepted Apr 21, 2021 \\
\hline
\end{tabular}

\section{Keywords:}

Device-to-device Stochastic geometry Outage probability

\begin{abstract}
Device-to-device (D2D) communication has been proposed to employ the proximity between two devices to enhance the overall spectrum utilization of a crowded cellular network. With the help of geometric probability tools, this framework considers the performance of cellular users under spatial separation with the D2D pair is investigated. The measurement results and analytical expression of outage probability show that the proposed frameworks improve the outage performance at a high signal-tonoise ratio (SNR) at the base station. Results also interpret that the distances between nodes in the D2D-assisted network make slight impacts on the performance of the cellular user.
\end{abstract}

Corresponding Author:

Dinh-Thuan Do

Faculty of Electronics Technology

Industrial University of Ho Chi Minh City

12 Nguyen Van Bao Road, Go Vap District, Ho Chi Minh City, Vietnam

Email: dodinhthuan@iuh.edu.vn

\section{INTRODUCTION}

Device to device (D2D) communication has recently been introduced in 3rd-generation partnership project (3GPP) long term evolution (LTE) Release 12 and 13. D2D, together with the core technologies, is proposed for forthcoming 5th generation $(5 \mathrm{G})$ cellular standard. In wireless cellular networks, the device-todevice (D2D) communications allow devices in close proximity to transmit and receive signals directly without connecting with base stations (BSs). Therefore, to reduce the high load from core networks, D2D has been recognized as a reasonable technique [1]-[3]. Together operation of the cellular network, the D2D devices are able to share the same spectrum resources with cellular users (CUs), and the spectral efficiency can be enhanced. In principle, D2D and cellular communication links are categorized into two kinds, including the overlay and underlay spectrum sharing techniques [4]. D2D users can only utilize the idle spectrum that is not currently employed by the CUs in the overlay D2D mode. The underlay spectrum sharing mode permits D2D pair users to occupy the resource of the cellular connections. The co-channel interference between the D2D and cellular links occurs, and such limitations need to be addressed. Therefore, the resource allocation problems are studied in D2D communications underlaying the cellular networks [5]-[7]. In [5], to maximize the sum rate of D2D links, the authors presented a power allocation scheme, and guarantee the performance of the cellular link at the same time. In order to maximize the rate of the single D2D link in [6], power allocation for D2D and cellular links is studied. The multiple cellular and D2D links are investigated in [7].

Considering the advantages of D2D communication, There are some studies on the usage of D2D communications in [8]-[12]. The cellular networks require high maintenance costs and additional infrastructure, and more difficulties with a large amount of equipment. In order to address these challenges, D2D communica- 
tions have been proposed as an essential technique to implement with non-orthogonal multiple access (NOMA) networks, the heterogeneous network recently [13]-[19].

To study a large number of nodes at various locations in D2D, stochastic geometry is a sophisticated tool from mathematics to provide insights into spatial averages taken over several realizations. Many metrics can be evaluated, such as outage probability, interference, data rate, and signal-to-interference-plus-noise ratio (SINR) [20],[21]. Stochastic geometry can be employed to design many applications related to D2D [22],[23]. Moreover, the published works [24]-[28] also study the performance of underlay D2D networks. The outage probability of random D2D communication in millimeter-wave cellular networks is studied in [24]. Moreover, the authors in [25] investigate the performance over nakagami- $m$ fading channels. The paper [26] also attempts to investigate the secure property of the D2D network. An interesting model where multiple D2D pairs are deployed is proposed in [27]. An extension to full-duplex communication for the D2D network is introduced by [28]. In the above works, there is no concern over the spatial separation between the cellular network and the D2D network. In addition, statistical properties of the distance between users in cellular networks and D2D networks under the nodal distancing constraint have not been reported in the literature.

Motivated by these analysis, this paper examines outage performance of traditional users located in outside of D2D serving area in cellular network.

\section{SYSTEM MODEL}

We consider a downlink wireless network in which there is a base station (BS), a cellular user (CUE) sharing resources with a D2D transmitter (D2D-Tx), as plotted in Figure 1. The CUE is uniformly distributed inside the cellular coverage area with radius $R_{c}$ around the BS. In addition, the CUE is located outside the D2D coverage area with radius $R_{d} \ll R_{c}$ around the D2D-Tx. We facilitate each node with a single antenna.

Further, the channel coefficients of the links from the BS and from the D2D-Tx to the CUE are denoted by $h$ and $g$, respectively. We assume all channels to be experiencing independent Rayleigh fading with $\mathbb{E}\left[|h|^{2}\right]=\mathbb{E}\left[|g|^{2}\right]=1$. To capture the large-scale propagation, we adopt the power-law path-loss model where $R$ and $L$ are the corresponding distances to the CUE, and $\eta$ denotes the pathloss exponent. Moreover, we assume a block-fading model in which all channels are invariant during the transmission of a block and vary independently among different blocks.

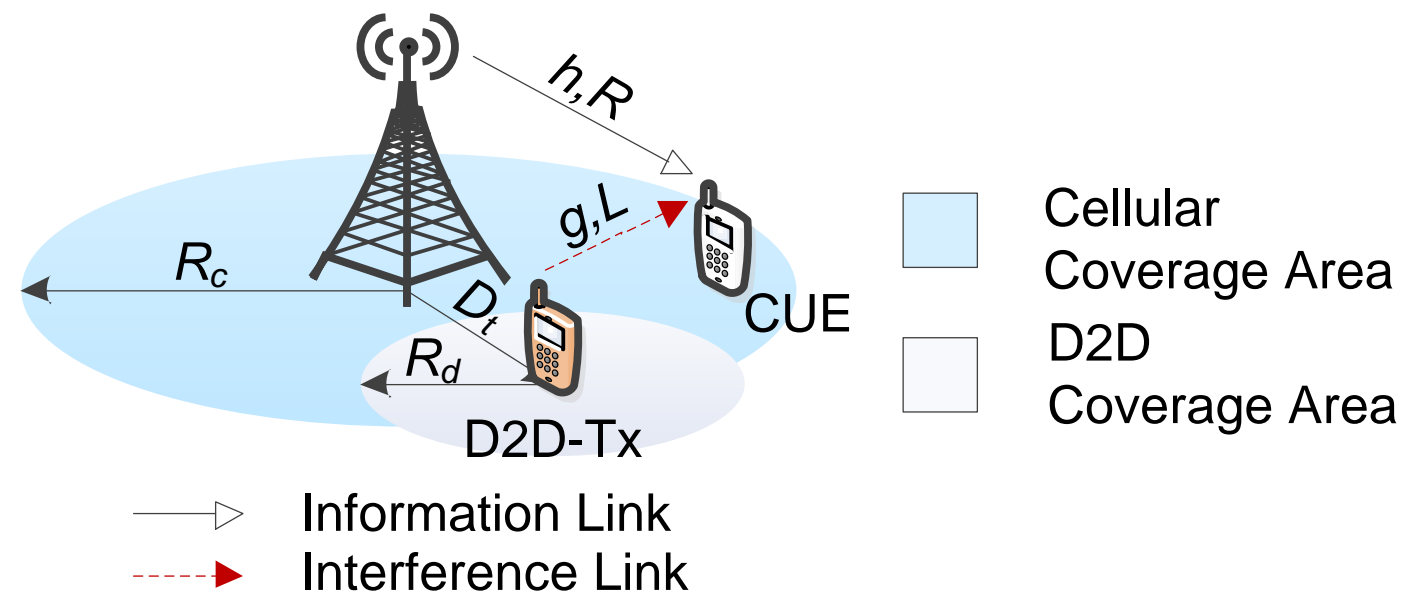

Figure 1. The proposed underlay D2D communication network.

In the downlink, the CUE receives the information signal from the BS while being interfered by the D2D-Tx. Subsequently, the received signal at the CUE is given by

$$
y=\sqrt{P_{b}} x \frac{h}{\sqrt{R^{\eta}}}+\sqrt{P_{t}} s \frac{g}{\sqrt{L^{\eta}}}+n
$$


in which $P_{b}$ and $P_{t}$ are the transmit powers from the BS and the D2D-Tx, respectively, $x$ and $s$ are the corresponding signals. Herein, $\mathbb{E}\left[|x|^{2}\right]=\mathbb{E}\left[|s|^{2}\right]=1$, and $n$ denotes the additive white Gaussian noise (AWGB) at the CUE with zero mean and variance $\sigma^{2}$. Hence, the achievable instantaneous rate at the given CUE is

$$
\mathcal{R}=\log _{2}\left(1+\frac{P_{b} \frac{|h|^{2}}{R^{\eta}}}{P_{t} \frac{|g|^{2}}{L^{\eta}}+\sigma^{2}}\right) .
$$

\section{DISTANCE DISTRIBUTION}

This section studies the stochastic properties of the link distances. Particularly, the CDF and PDF of the distances $R$ and $L$ are derived in closed-form. It should be mentioned that both $L$ and $R$ are dependent on the location of the CUE.

\subsection{Preliminaries}

In this subsection, we specify the distribution, i.e., CDF and PDF, of the distance from a fixed point $A$ to the random point $C$. Particularly, the point $C$ uniformly distributed inside the circular area with the radius $R$ around a fixed point $B$, i.e., $C \in \mathcal{C}(B, R)$. The distance probability distribution function is considered in two different cases as depicted in [29, Eq.(34)], and can be formulated as

$$
F_{A C}(A C)=\frac{|\mathcal{C}(A, A C) \cap \mathcal{C}(B, R)|}{|\mathcal{C}(B, R)|}, A B-R<A C<A B+R
$$

in which $|\cdot|$ denotes the Lebesgue measure. Denoting $A_{T O T} \triangleq \mathcal{C}(A, A C) \cap \mathcal{C}(B, R)$, for the case $A B \geq R$, $\left|A_{T O T}\right|$ is calculated as by [29]

$$
\begin{aligned}
\left|A_{\text {TOT }}\right|= & \frac{\pi}{2}\left(A C^{2}+R^{2}\right)-A C^{2} \sin ^{-1}\left(\frac{A B^{2}+A C^{2}-R^{2}}{2 A C \cdot A B}\right)+R^{2} \sin ^{-1}\left(\frac{A C^{2}-A B^{2}-R^{2}}{2 R . A B}\right) \\
& -\frac{1}{2} \sqrt{\left(A C^{2}-(A B-R)^{2}\right)\left((A B+R)^{2}-A C^{2}\right)} \triangleq S(A B, R ; A C),
\end{aligned}
$$

with $A B-R \leq A C \leq A B+R$. For the case $A B \leq R$, the area of $A_{T O T}$ is obtained as [29]

$$
\left|A_{T O T}\right|= \begin{cases}\pi A C^{2} & , 0 \leq A C \leq R-A B \\ S(A B, R ; A C) & , R-A B \leq A C \leq R+A B\end{cases}
$$

\subsection{The information links}

Initially, let $\mathcal{A}_{C}$ be the 2D-Euclidean space in which the CUE uniformly distributed and $\mathcal{A}_{D}$ be the coverage area around the D2D-Tx. To reduce the cross-mode interference, the interference from the D2D-Tx to the CUE and vice versa, it is assumed that $\mathcal{A}_{C}$ and $\mathcal{A}_{D}$ are disjoint meaning that the CUE is located outside the D2D proximity. Hence, the CDF of $R$ is given as

$$
F_{R}(r)=\frac{\left|\mathcal{C}(\mathrm{BS}, r) \backslash \mathcal{A}_{D}\right|}{\left|\mathcal{A}_{C}\right|}, 0<r<R_{c} .
$$

Denoting $\mathcal{S} \triangleq \mathcal{C}(\mathrm{BS}, r) \backslash \mathcal{A}_{D}$, the above expression can further be derived via the four following cases. a) Case $D_{t}+R_{d} \leq R_{c} \wedge D_{t} \geq R_{d}$ :

As shown in Figure 2, $\left|\mathcal{A}_{C}\right|=\pi R_{c}^{2}-\pi R_{d}^{2}$ and the calculation for $|\mathcal{S}|$ is divided into three following subcases: 1) if $0<r<D_{t}-R_{d}$, it is clearly that $\left.|\mathcal{S}|=\pi r^{2}, 2\right)$ if $D_{R}+R_{d}<r<R_{c},|\mathcal{S}|=\pi r^{2}-\pi R_{d}^{2}$ and 3) if $D_{t}-R_{d}<r<D_{t}+R_{d}$, thus

$$
|\mathcal{S}|=|\mathcal{C}(\mathrm{BS}, r)|-\left|\mathcal{C}(\mathrm{BS}, r) \cap \mathcal{A}_{D}\right|=\pi r^{2}-S\left(D_{t}, R_{d} ; r\right) .
$$

Now, the corresponding PDF is obtained by substituting (7) into (6) and then taking the derivative of the resulted $F_{R}(r)$, thus

$$
f_{R}(r)=\frac{1}{\pi R_{c}^{2}-\pi R_{d}^{2}} \begin{cases}2 \pi r & , 0<r<D_{t}-R_{d} \\ \pi r+2 r \sin ^{-1}\left(\frac{D_{t}^{2}+r^{2}-R_{d}^{2}}{2 D_{t} r}\right) & , D_{t}-R_{d}<r<D_{t}+R_{d} \\ 2 \pi r & , D_{t}+R_{d}<r<R_{c}\end{cases}
$$


Note that [29]

$$
\frac{\partial}{\partial A C} S(A B, R ; A C)=\pi A C-2 A C \sin ^{-1}\left(\frac{A B^{2}+A C^{2}-R^{2}}{2 A B \cdot R}\right) .
$$

b) Case $D_{t}+R_{d} \leq R_{c} \wedge D_{t} \leq R_{d}$ :

As shown in Figure 3, $\left|\mathcal{A}_{C}\right|=\pi R_{c}^{2}-\pi R_{d}^{2}$ and $|\mathcal{S}|$ is derived as following subcases: 1) if $0<r<$ $R_{d}-D_{t}$, thus $\left.|\mathcal{S}|=0,2\right)$ if $D_{t}+R_{d}<r<R_{c}$, thus $|\mathcal{S}|=\pi r^{2}-\pi R_{d}^{2}$, and 3) if $D_{t}-R_{d}<r<D_{t}+R_{d}$, $|\mathcal{S}|=\pi r^{2}-S\left(D_{t}, R_{d} ; r\right)$.

As a result, the corresponding PDF is given by

$$
f_{R}(r)=\frac{1}{\pi R_{c}^{2}-\pi R_{d}^{2}} \begin{cases}0 & , 0<r<R_{d}-D_{t} \\ \pi r+2 r \sin ^{-1}\left(\frac{D_{t}^{2}+r^{2}-R_{d}^{2}}{2 D_{t} r}\right) & , D_{t}-R_{d}<r<D_{t}+R_{d} \\ 2 \pi r & , D_{t}+R_{d}<r<R_{c}\end{cases}
$$

c) Case $D_{t}+R_{d} \geq R_{c} \geq D_{t}-R_{d}$ :

The cellular coverage is given as $\left|\mathcal{A}_{C}\right|=\pi R_{c}^{2}-|\mathcal{A}|$ where $\mathcal{A}=\mathcal{C}\left(\mathrm{BS}, R_{c}\right) \cap \mathcal{A}_{D}$ as shown in Figure 4. It is known that [30]

$$
\begin{aligned}
|\mathcal{A}|= & R_{d}^{2} \cos ^{-1}\left(\frac{D_{t}^{2}+R_{d}^{2}-R_{c}^{2}}{2 D_{t} R_{d}}\right)+R_{c}^{2} \cos ^{-1}\left(\frac{D_{t}^{2}+R_{c}^{2}-R_{d}^{2}}{2 D_{t} R_{c}}\right) \\
& -\frac{1}{2} \sqrt{\left(-D_{t}+R_{d}+R_{c}\right)\left(D_{t}+R_{d}-R_{c}\right)\left(D_{t}-R_{d}+R_{c}\right)\left(D_{t}+R_{d}+R_{c}\right)} .
\end{aligned}
$$

In this case, $|\mathcal{S}|$ can be derived via the two following subcases: 1) if $0<r<R_{d}-D_{t}$, thus $|\mathcal{S}|=\pi r^{2}$, and 2) if $D_{t}-R_{d}<r<D_{t}+R_{d},|\mathcal{S}|=\pi r^{2}-S\left(D_{t}, R_{d} ; r\right)$.

Hence, the PDF of the distance of the information link is then obtained as

$$
f_{R}(r)=\frac{1}{\pi R_{c}^{2}-|\mathcal{A}|} \begin{cases}2 \pi r & , 0<r<D_{t}-R_{d} \\ \pi r+2 r \sin ^{-1}\left(\frac{D_{t}^{2}+r^{2}-R_{d}^{2}}{2 D_{t} r}\right) & , D_{t}-R_{d}<r<R_{c}\end{cases}
$$

d) Case $D_{t}-R_{d} \geq R_{c}$ :

In this case, $\mathcal{C}\left(\mathrm{BS}, R_{c}\right) \cap \mathcal{A}_{D}=\emptyset$, thus the $\mathrm{CDF}$ and the PDF of the distance $R$ are immediately given as

$$
\begin{aligned}
& F_{R}(r)=\frac{r^{2}}{R_{c}^{2}}, 0<r<R_{c}, \\
& f_{R}(r)=\frac{2 r}{R_{c}^{2}}, 0<r<R_{c} .
\end{aligned}
$$

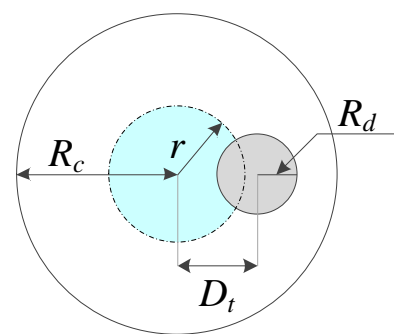

Figure 2. A realization of $D_{t}+R_{d} \leq R_{c} \wedge D_{t} \geq R_{d}$

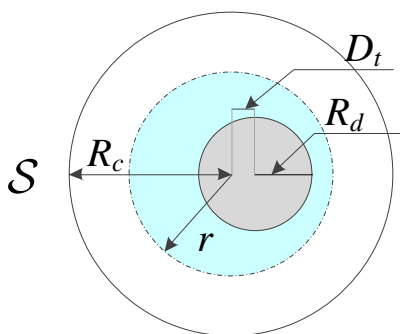

Figure 3. A realization of $D_{t}+R_{d} \leq R_{c} \wedge D_{t} \leq R_{d}$

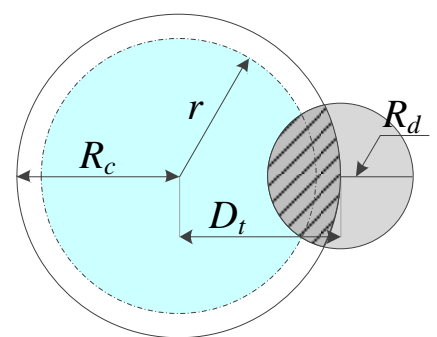

$\mathcal{S}$ $\mathcal{A}$

Figure 4. A realization of $D_{t}+R_{d} \geq R_{c} \geq D_{t}-R_{d}$ 


\subsection{The interference links}

In this subsection, the CDF and PDF of the link distance from the interfering D2D-Tx to the CUE are studied. Similar to the previous parts, the CDF of the distance $L$ is given as

$$
F_{L}(r)=\frac{\left|\mathcal{A}_{C} \cap \mathcal{C}(\mathrm{D} 2 \mathrm{D}-\mathrm{Tx}, r)\right|}{\left|\mathcal{A}_{C}\right|}, 0<r<D_{t}+R_{d}
$$

Note that we reuse the notation $\mathcal{S} \triangleq \mathcal{A}_{C} \cap \mathcal{C}(\mathrm{D} 2 \mathrm{D}-\mathrm{Tx}, r)$ for the following analysis. Subsequently, the above expression can further be derived via the three following cases.

a) Case $D_{t}+R_{d} \leq R_{c}$ :

Similarly, $\left|\mathcal{A}_{C}\right|=\pi R_{c}^{2}-\pi R_{d}^{2}$ and $|\mathcal{S}|$ is derived by the following subcases. 1) if $r<R_{d}$, it is obvious that $|\mathcal{S}|=0,2)$ if $R_{d}<r<R_{c}-D_{t},|\mathcal{S}|=\pi r^{2}-\pi R_{d}^{2}$, and 3) if $R_{c}-D_{t}<r<R_{c}+D_{t}$, $|\mathcal{S}|=S\left(D_{t}, R_{c} ; r\right)-\pi R_{d}^{2}$. Subsequently, the PDF of the interference distance is then given as

$$
f_{L}(r)=\frac{1}{\pi R_{c}^{2}-\pi R_{d}^{2}} \begin{cases}0 & , 0<r<R_{d} \\ 2 \pi r & , R_{d}<r<R_{c}-D_{t} \\ \pi r-2 r \sin ^{-1}\left(\frac{D_{t}^{2}+r^{2}-R_{c}^{2}}{2 D_{t} r}\right) & , R_{c}-D_{t}<r<R_{c}+D_{t}\end{cases}
$$

b) Case $D_{t}+R_{d} \geq R_{c} \geq D_{t}-R_{d}$ :

Similar to the previous subsection, $\left|\mathcal{A}_{C}\right|=\pi R_{c}^{2}-|\mathcal{A}|$ and $|\mathcal{S}|=S\left(D_{t}, R_{d} ; r\right)-|\mathcal{A}|$ if $R_{d}<r<$ $R_{d}+D_{t}$ whereas $|\mathcal{S}|=0$ whenever $r<R_{d}$. Hence,

$$
f_{L}(r)=\frac{1}{\pi R_{c}^{2}-|\mathcal{A}|} \begin{cases}0 & , 0<r<R_{d} \\ \pi r-2 r \sin ^{-1}\left(\frac{D_{t}^{2}+r^{2}-R_{c}^{2}}{2 D_{t} r}\right) & , R_{d}<r<R_{c}+D_{t}\end{cases}
$$

c) Case $D_{t}-R_{d} \geq R_{c}$ :

In this case, $\left|\mathcal{A}_{C}\right|=\pi R_{c}^{2}$ and $|\mathcal{S}|$ is obtained as [...]

$$
|\mathcal{S}|= \begin{cases}S\left(D_{t}, R_{c} ; r\right) & , D_{t}-R_{c}<r<D_{t}+R_{c} \\ 0 & , 0<r<D_{t}-R_{c}\end{cases}
$$

Substituting the above result into (15) and with the help of (9), one can the obtain

$$
f_{L}(r)=\frac{1}{\pi R_{c}^{2}} \begin{cases}\pi r-2 r \sin ^{-1}\left(\frac{D_{t}^{2}+r^{2}-R_{c}^{2}}{2 D_{t} r}\right) & , D_{t}-R_{c}<r<D_{t}+R_{c} \\ 0 & , 0<r<D_{t}-R_{c}\end{cases}
$$

\section{OUTAGE PERFORMANCE}

In this section, we investigate the outage probability of the proposed system. Let $R_{t h}$ be the target data rate of the CUE, thus the probability for the instantaneous rate at this CUE falls below $R_{t h}$ is mathematically expressed as

$$
\begin{aligned}
P_{\text {out }} & =\operatorname{Pr}\left\{\mathcal{R}<\bar{R}_{t h}\right\} \\
& =1-\operatorname{Pr}\left\{|h|^{2} \geq \frac{R^{\eta} \bar{\gamma}_{t h}}{\bar{\gamma}_{b}}\left(\frac{\bar{\gamma}_{t}|g|^{2}}{L^{\eta}}+1\right)\right\},
\end{aligned}
$$

in which $\bar{\gamma}_{t h} \triangleq 2^{R_{t h}}-1, \bar{\gamma}_{b} \triangleq P_{b} / \sigma^{2}$ and $\bar{\gamma}_{t} \triangleq P_{t} / \sigma^{2}$. Now that $|h|$ and $|g|$ are both independent and identical Rayleigh distributed random variables with unit variance, thus the CDF of $|h|^{2}$ and the PDF of $|g|^{2}$ are given by

$$
\begin{gathered}
F_{|h|^{2}}(x)=1-e^{-x}, x>0, \\
f_{|g|^{2}}(x)=e^{-x}, x>0
\end{gathered}
$$


respectively. Hence (21) can further be expressed as

$$
\begin{aligned}
P_{\text {out }} & =1-\mathbb{E}_{R,|g|^{2}, L}\left[\exp \left\{-\frac{R^{\eta} \bar{\gamma}_{t h}}{\bar{\gamma}_{b}}\left(\frac{\bar{\gamma}_{t}|g|^{2}}{L^{\eta}}+1\right)\right\}\right] \\
& \approx 1-\int_{0}^{R_{c}} \exp \left(-\frac{r^{\eta} \bar{\gamma}_{t h}}{\bar{\gamma}_{b}}\left(\bar{\gamma}_{t} \bar{g}+1\right)\right) f_{R}(r) d r,
\end{aligned}
$$

in which (23) is proposed to approximate (22). The tightness of this approximation will be verified later in the next section. Herein, $\bar{g}$ denotes the mean interference channel power gain at the CUE and is calculated as

$$
\bar{g} \triangleq \mathbb{E}\left[|g|^{2} L^{-\eta}\right]=\int_{0}^{D_{t}+R_{c}} \frac{f_{L}(l)}{l^{\eta}} d l .
$$

\section{NUMERICAL RESULTS}

In this section, Monte Carlo simulations are constrcuted to validate our analysis in the previous sections. The default settings for the results are $R_{c}=15(\mathrm{~m}), R_{d}=5(\mathrm{~m}), R_{t h}=0.5(\mathrm{bps} / \mathrm{Hz}), \bar{\gamma}_{t}=10(\mathrm{~dB})$ and $\eta=2$. In addition, the average transmit SNR is set to $\bar{\gamma}_{b}=30(d B)$ in the Figure 6 .

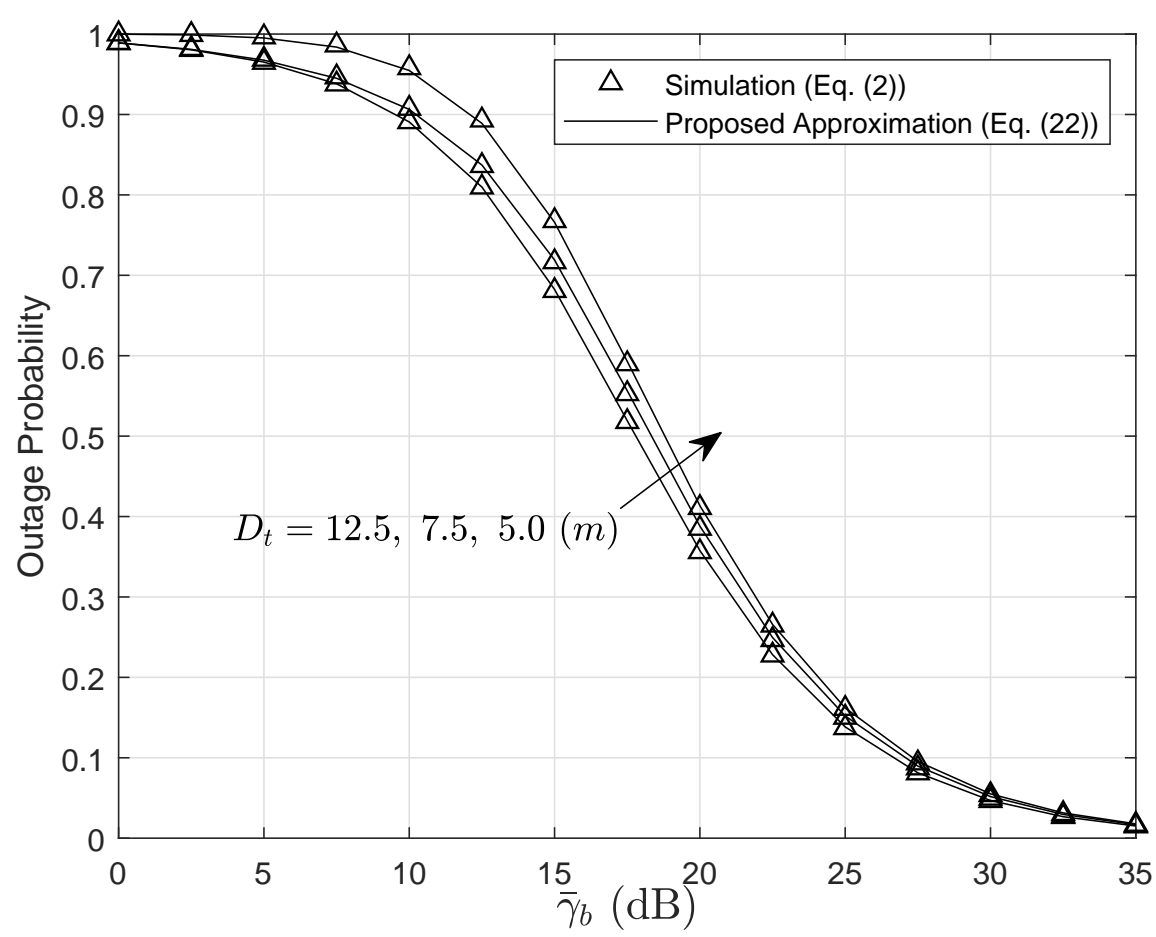

Figure 5. Outage probability at the CUE versus the average transmit signal-to-noise of the BS $\left(\bar{\gamma}_{b}\right)$ and the distance $D_{t}(m)$

Figure 5 shows the outage performance comparison of conventional user as changing $D_{t}$. It can be seen that as varying $D_{t}$ from $5(\mathrm{~m})$ to $12.5(\mathrm{~m})$, a small performance gap emerges. Increasing transmit SNR at the BS contributes to improving outage performance. It is confirmed that analytical and simulation results are matched very tight at the whole range of SNR. Hence, it can be believed that the proposed approximation acceptably tight. Figure 6 further evaluates the mean interference channel power gain of the considered system to validate our exact analysis for the link distance distribution. 


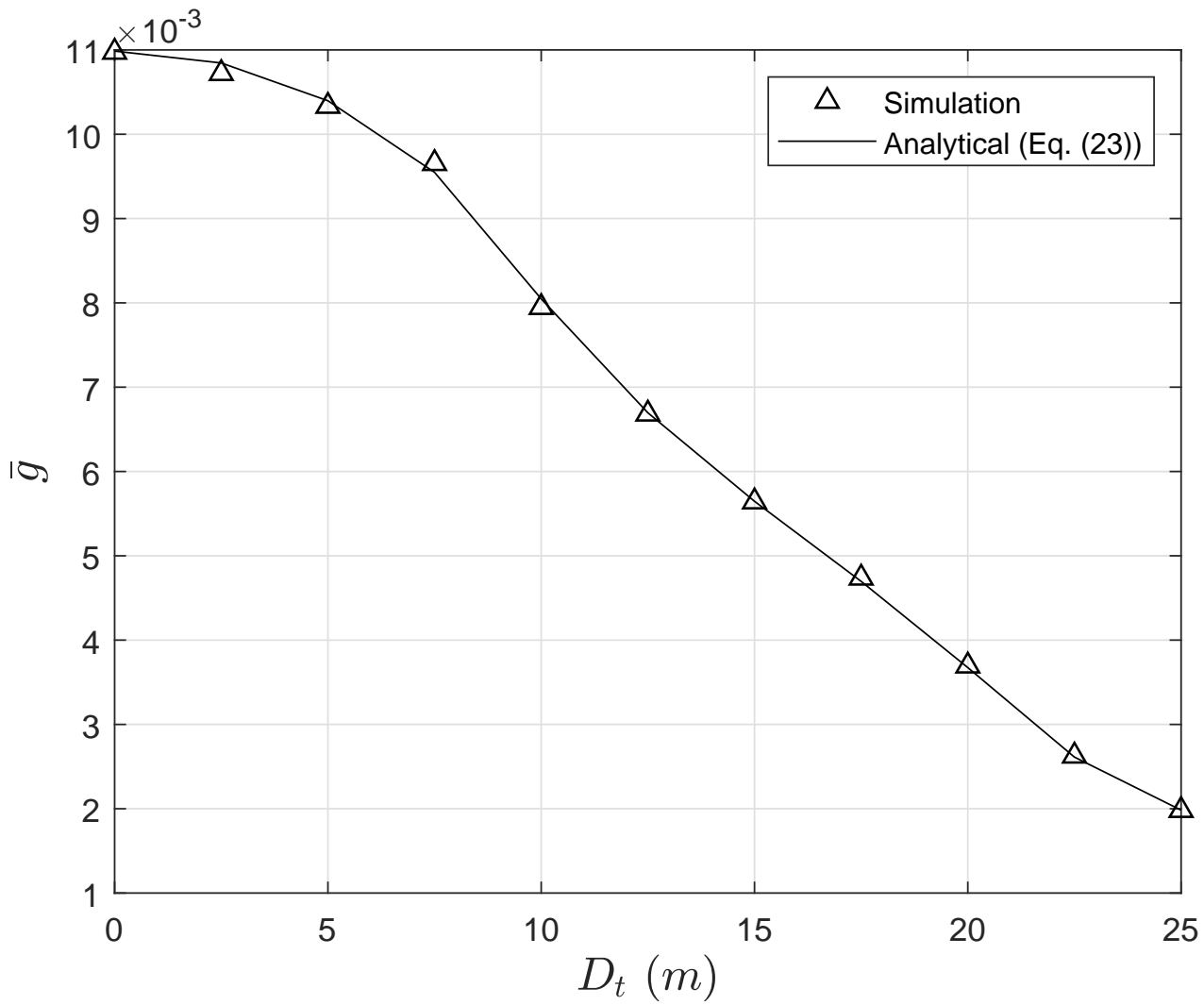

Figure 6. Mean interference channel power gain at the CUE versus the distance $D_{t}(\mathrm{~m})$

\section{CONCLUSION}

In this paper, we have studied the outage performance problem of a cellular user in the context of the D2D underlying cellular network. The outage probability of users in D2D communication is computed. Specifically, the distance from the cellular user to the BS and from the D2D transmitter to the user are both statistically characterized using stochastic geometry tools. We also derived the exact and approximated expressions of outage probability at the cellular node. Numerical results are provided to verify our derivations. The paper is, however, limited at downlink single-cell networks, and will be extended to the multi-cell sencario in our future works.

\section{REFERENCES}

[1] K. Doppler, M. Rinne, C. Wijting, C. Ribeiro, and K. Hugl, "Device-todevice communication as an underlay to LTE-advanced networks," IEEE Commun. Mag., vol. 47, no. 12, pp. 42-49, Dec. 2009.

[2] G. Fodor et al., "Design aspects of network assisted device-to-device communications," IEEE Commun. Mag., vol. 50, no. 3, pp. 170-177, Mar. 2012.

[3] J. Liu, Y. Kawamoto, H. Nishiyama, N. Kato, and N. Kadowaki, "Device-to-device communications achieve efficient load balancing in LTE-advanced networks," IEEE Wirel. Commun. Mag., vol. 21, no. 2, pp. 57-65, Feb. 2014.

[4] X. Lin, J. G. Andrews, and A. Ghosh, "Spectrum sharing for deviceto-device communication in cellular networks," IEEE Trans. Wirel. Commun., vol. 13, no. 12, pp. 6727-6740, Dec. 2014.

[5] C.-H. Yu, O. Tirkkonen, K. Doppler, and C. Ribeiro, "On the performance of device-to-device underlay communication with simple power control," Proc. VTC, Apr. 2009, pp. 1-5.

[6] J. Wang, D. Zhu, C. Zhao, J. C. F. Li, and M. Lei, "Resource sharing of underlaying device-to-device and uplink cellular communications," IEEE Commun. Lett., vol. 17, no. 6, pp. 1148-1151, Jun. 2013.

[7] D. Feng, L. Lu, Y. Yuan-Wu, G. Y. Li, G. Feng, and S. Li, ”Deviceto-device communications underlaying cellular networks," IEEE Trans. Commun., vol. 61, no. 8, pp. 3541-3551, Aug. 2013. 
[8] J. Liu, N. Kato, J. Ma, and N. Kadowaki, "Device-to-device communication in LTE-advanced networks: A survey," IEEE Commun. Surv. Tuts., vol. 17, no. 4, pp. 1923-1940, 4th Quart., 2015.

[9] F. Jameel, Z. Hamid, F. Jabeen, S. Zeadally, and M. A. Javed, "A survey of device-to-device communications: Research issues and challenges," IEEE Commun. Surv. Tuts., vol. 20, no. 3, pp. 2133-2168, 3rd Quart., 2018.

[10] P. Gandotra and R. K. Jha, "Device-to-device communication in cellular networks: A survey," J. Netw. Comput. Appl., vol. 71, pp. 99-117, Aug. 2016.

[11] H. A. U. Mustafa, M. A. Imran, M. Z. Shakir, A. Imran, and R. Tafazolli, "Separation framework: An enabler for cooperative and D2D communication for future 5G networks," IEEE Commun. Surv. Tuts., vol. 18, no. 1, pp. 419-445, 1st Quart., 2016.

[12] M. Höyhtyä, O. Apilo, and M. Lasanen, "Review of latest advances in 3GPP standardization: D2D communication in 5G systems and its energy consumption models," Future Internet, vol. 10, no. 1, p. 3, 2018.

[13] D. Feng et al., "Mode switching for energy-efficient device-to-device communications in cellular networks," IEEE Trans. Wirel. Commun., vol. 14, no. 12, pp. 6993-7003, Dec. 2015.

[14] X. Lin, J. G. Andrews, and A. Ghosh, "Spectrum sharing for deviceto-device communication in cellular networks," IEEE Trans. Wirel. Commun., vol. 13, no. 12, pp. 6727-6740, Dec. 2014.

[15] L. Zhang, M. Xiao, G. Wu, and S. Li, "Efficient scheduling and power allocation for D2D-assisted wireless caching networks," IEEE J. Sel. Areas Commun., vol. 64, no. 6, pp. 2438-2452, Jun. 2016.

[16] S. Xu, H. Zhang, J. Tian, and P. T. Mathiopoulos, "Pilot reuse and power control of D2D underlaying massive MIMO systems for energy efficiency optimization," Sci. China Inf. Sci., vol. 60, no. 10, 2017, Art. no. 100303.

[17] Dinh-Thuan Do, A.-T. Le, C.-B. Le and B. M. Lee, "On Exact Outage and Throughput Performance of Cognitive Radio based Non-Orthogonal Multiple Access Networks With and Without D2D Link," Sensors, 19 (15): 3314, 2019.

[18] C.-B. Le and Dinh-Thuan Do, "Joint Evaluation of Imperfect SIC and Fixed Power Allocation Scheme for Wireless Powered D2D-NOMA networks with multiple antennas at Base Station," Wireless Networks, vol. 25, no. 8, pp. 5069-5081, 2019

[19] H.P.-Dang, Dinh-Thuan Do, et al, "Power Beacon-based Wireless Power Transfer in MISO/SISO: An application in Device-to-Device networks," Wireless Personal Communications (Springer), Vol. , No. , pp. , 2019.

[20] M. Haenggi, J. G. Andrews, F. Baccelli, O. Dousse, and M. Franceschetti, "Stochastic geometry and random graphs for the analysis and design of wireless networks," IEEE J. Sel. Areas Commun., vol. 27, no. 7, pp. 1029-1046, Sep. 2009.

[21] H. Elsawy, E. Hossain, and M. Haenggi, "Stochastic geometry for modeling, analysis, and design of multi-tier and cognitive cellular wireless networks: A survey," IEEE Commun. Surv. Tuts., vol. 15, no. 3, pp. 996-1019, Jun. 2013.

[22] O. A. Amodu, M. Othman, N. K. Noordin and I. Ahmad, "Relay-Assisted D2D Underlay Cellular Network Analysis Using Stochastic Geometry: Overview and Future Directions," IEEE Access, vol. 7, pp. 115023-115051, 2019.

[23] D. Singh and S. C. Ghosh, "Mobility-Aware Relay Selection in 5G D2D Communication Using Stochastic Model," IEEE Trans. Veh. Technol., vol. 68, no. 3, pp. 2837-2849, March 2019.

[24] S. Kusaladharma, Z. Zhang and C. Tellambura, "Interference and Outage Analysis of Random D2D Networks Underlaying Millimeter-Wave Cellular Networks," IEEE Trans. Commun., vol. 67, no. 1, pp. 778-790, Jan. 2019.

[25] I. Singh, R. K. Jaiswal, V. Kumar, R. Verma, N. P. Singh and G. Singh, ”Outage Probability of Device-to-Device Communication Underlaying Cellular Network over NakagamiRayleigh Fading Channels," 2019, 9th International Conference on Emerging Trends in Engineering and Technology - Signal and Information Processing (ICETET-SIP19), Nagpur, India, 2019, pp. 1-5.

[26] J. Lyu, T. Zheng, K. Huang, Y. Feng and H. Wang, "Secure Transmissions of D2D Underlay Cellular Networks with Poisson Cluster Process," 2019, IEEE Global Communications Conference (GLOBECOM), Waikoloa, HI, USA, 2019, pp. 1-6.

[27] J. Lee and J. H. Lee, "Performance Analysis and Resource Allocation for Cooperative D2D Communication in Cellular Networks With Multiple D2D Pairs," IEEE Commun. Lett., vol. 23, no. 5, pp. 909-912, May 2019.

[28] G. Liu, W. Feng, Z. Han and W. Jiang, "Performance Analysis and Optimization of Cooperative Full-Duplex D2D Communication Underlaying Cellular Networks," IEEE Trans. Wirel. Commun, vol. 18, no. 11, pp. 5113-5127, Nov. 2019.

[29] F. Adelantado, J. Prez-Romero, and O. Sallent, "Nonuniform traffic distribution model in reverse link of multirate/multiservice wcdma-based systems," IEEE Trans. Veh. Technol., vol. 56, no. 5, p. 29022914, Sep. 2007.

[30] Weisstein, Eric W. "Circle-Circle Intersection." From MathWorld-A Wolfram Web Resource. http://mathworld.wolfram.com/Circle-CircleIntersection.html 